\title{
Research update on the anticancer effects of buparlisib (Review)
}

\author{
JINSHAN XING ${ }^{1}$, JUN YANG ${ }^{1}$, YINGJIANG GU ${ }^{1}$ and JINGYAN YI ${ }^{2}$ \\ ${ }^{1}$ Department of Neurosurgery, The Affiliated Traditional Chinese Medicine Hospital of Southwest Medical University; \\ ${ }^{2}$ Department of Medical Cell Biology and Genetics, School of Basic Medical Sciences, \\ Southwest Medical University, Luzhou, Sichuan 646000, P.R. China
}

Received October 16, 2020; Accepted January 18, 2021

DOI: 10.3892/ol.2021.12527

\begin{abstract}
Buparlisib is a highly efficient and selective PI3K inhibitor and a member of the 2,6-dimorpholinopyrimidine-derived family of compounds. It selectively inhibits four isomers of PI3K, PI3K $\alpha, \mathrm{PI} 3 \mathrm{~K} \beta, \mathrm{PI} 3 \mathrm{~K} \gamma$ and $\mathrm{PI} 3 \mathrm{~K} \delta$, by competitively binding the lipid kinase domain on adenosine 5 '-triphosphate (ATP), and serves an important role in inhibiting proliferation, promoting apoptosis and blocking angiogenesis, predominantly by antagonizing the PI3K/AKT pathway. Buparlisib has been confirmed to have a clinical effect in patients with solid tumors and hematological malignancies. A global, phase II clinical trial with buparlisib and paclitaxel in head and neck squamous cell carcinoma has now been completed, with a manageable safety profile. Buparlisib currently has fast-track status with the United States Food and Drug Administration. The present review examined the biochemical structure, pharmacokinetic characteristics, preclinical data and ongoing clinical studies of buparlisib. The various mechanisms of influence of buparlisib in tumors, particularly in preclinical research, were summarized, providing a theoretical basis and direction for basic research on and clinical treatment with buparlisib.
\end{abstract}

\section{Contents}

1. Introduction

2. Pan-PI3K inhibitors

Correspondence to: Dr Jingyan Yi, Department of Medical Cell Biology and Genetics, School of Basic Medical Sciences, Southwest Medical University, Section 1, 1 Xianglin Road, Longmatan, Luzhou, Sichuan 646000, P.R. China

E-mail: jingyany@swmu.edu.cn

Professor Yingjiang Gu, Department of Neurosurgery, The Affiliated Traditional Chinese Medicine Hospital of Southwest Medical University, 182 Chunhui Road, Longmatan, Luzhou, Sichuan 646000, P.R. China

E-mail: lzmcgyj@126.com

Key words: PI3K, buparlisib, cancer, apoptosis, drug combination
3. Biochemical properties of buparlisib

4. Pharmacokinetic characteristics of buparlisib

5. Preclinical research on buparlisib

6. Ongoing clinical trials on buparlisib

7. Conclusion

\section{Introduction}

PI3K is an evolutionarily conserved lipid kinase family, with a dual activity (serine/threonine and phosphatidylinositol kinase activities). It interacts with receptors on the cell membrane and mediates the transmission of external signals into the cells. According to their protein domain structure and lipid substrate specificity, they are divided into three categories, each with different functions and mechanisms, and participate in the regulation of several intracellular processes $(1,2)$.

Class I includes heterodimer proteins, which mainly exist in the cytoplasm. These can be further systematically divided into IA and IB. Class IA is mainly activated by tyrosine kinase receptor, which is composed of catalytic subunit p110 and regulatory subunit p85 (3). The catalytic subunit p110 includes $\mathrm{p} 110 \alpha, \mathrm{p} 110 \beta$ and $\mathrm{p} 110 \delta$, which are encoded by PIK3CA, PIK3CB and PIK3CD, respectively (4). The regulatory subunit P85 includes p $85 \alpha, \mathrm{p} 85 \beta, \mathrm{p} 55 \alpha, \mathrm{p} 55 \gamma$ and $\mathrm{p} 50 \alpha(5)$. The binding of the catalytic and regulatory subunits has a dual activity (lipid and protein kinase activities). The IB class includes catalytic subunit p110 $\gamma$ and regulatory subunits p101, p84 and p87, which are mainly activated by upstream G protein-coupled receptors $(6,7)$. Class II consists of the $\mathrm{C} 2 \alpha$, $\mathrm{C} 2 \beta$ and $\mathrm{C} 2 \gamma$ subunits, including the PI3KC2a, PI3KC2b and PI3KC2c subunits, and appears to have an important role in insulin signal transduction, neuronal survival, growth factor signal transduction and angiogenesis (8). Class III PI3K includes Vps34-VPS15 heterodimers, which are involved in a variety of intracellular transport activities, including protein synthesis and autophagy (3). Compared with class II and class III, class I is the most widely studied. Studies have shown that these kinases are closely associated with tumor occurrence and development, growth and metabolism, and tumor microenvironment $(2,4,5)$. In mammals, four subtypes of p110 exist, of which the $110 \alpha$ and $p 110 \beta$ catalytic subunits are widely distributed in the majority of cell types, while p110 $\gamma$ and p110 are significantly enriched in immune cells (9). Several mutations in the PIK3CA gene encoding the p110 $\alpha$ subunit 
have been found in various types of cancer, including breast cancer, medulloblastoma and hepatocellular carcinoma $(4,5)$. In breast cancer, $>85 \%$ of the mutations in the PI3KCA gene occur in E542K and E545K, which encode helical domains, and H1047R, which encodes catalytic domains $(10,11)$.

Following ligand receptor activation, class I PI3K phosphorylates phosphatidylinositol 4,5-bisphosphate (PIP2), resulting in phosphatidylinositol 3,4,5-trisphosphate (PIP3) (12-15). It should be noted that tumour suppressor PTEN can hydrolyse PIP3 and convert it into PIP2, a negative regulator of the PI3K signalling pathway $(16,17)$. PIP3 is often referred to as the second messenger. Its pleckstrin homology domain can bind with 3-phosphoinositide-dependent kinase-1 (PDK1) and AKT and recruit them to the cell membrane $(13,18)$. At that time point, AKT has been shown to be activated by PDK1 through the phosphorylation of threonine 308 and serine 473 (19). mTOR is one of the downstream effectors of AKT. mTOR complex (mTORC) 1 and mTORC2 have different structures and functions. AKT mainly affects mTORC1, when it phosphorylates and activates its downstream ribosomal protein S6 kinase $\beta-1$ (p70S6K) and eukaryotic translation initiation factor 4E-binding protein 1 (4E-BP1), which leads to cell cycle regulation and increased ribosomal protein translation and synthesis, a process that stimulates a series of major drivers of cell growth, proliferation and angiogenesis $(20,21)$. mTORC2 regulates ion transport and cytoskeleton morphology, among other processes, through serine/threonine-protein kinase, protein kinase $\mathrm{C} \alpha$ and RAS $(22,23)$. In addition, mTORC2 is directly regulated by $\mathrm{PI} 3 \mathrm{~K}$ and has a feedback regulation on AKT.

The uncontrolled activation of the PI3K/AKT/mTOR pathway, which is closely associated with several types of cancer, is involved in tumorigenesis, proliferation, invasion, cell cycle progression, apoptosis, metastasis and chemotherapy resistance $(2,24,25)$. Recent studies have shown that the PI3K/AKT/mTOR pathway is also associated with angiogenesis, cytoskeleton, inflammatory response and oxidative stress (26-28). This pathway has a wide range of physiological and pathological functions, and is one of the most important oncogenic pathways in human cancer. At present, PI3K, AKT, mTORC1/2 and mTOR/PI3K inhibitors are the most widely used inhibitors in treatment targeting the PI3K/AKT/mTOR pathway. PI3K inhibitors include pan-PI3K inhibitors, targeting all four PI3K class I subtypes (PI3K $\alpha, \mathrm{PI} 3 \mathrm{~K} \beta, \mathrm{PI} 3 \mathrm{~K} \gamma$ and PI3Kס), as well as allosteric PI3K inhibitors, which are specific to one isomer (29). Buparlisib is a pan-PI3K inhibitor.

The present review summarized the biochemical structure, pharmacokinetic characteristics, preclinical data and ongoing clinical studies of buparlisib. Particularly in preclinical research, the present article reviewed the current knowledge on various molecular factors and signaling pathways affected by buparlisib, which may provide a theoretical basis and direction for basic research and clinical treatment of cancer involving buparlisib.

\section{Pan-PI3K inhibitors}

Pan-PI3K inhibitors have a wide range of activities against class I PI3K subtypes (PI3K $\alpha, \mathrm{PI} 3 \mathrm{~K} \beta, \mathrm{PI} 3 \mathrm{~K} \gamma$ and PI3Kס). Several pan-PI3K inhibitors have entered clinical trials.
Copanlisib (BAY80-6946). Copanlisib was developed by Bayer AG and approved for listing by the United States Food and Drug Administration (FDA) in 2017. Its trade name is Aliqopa. Copanlisib, which acts on PI3K $\alpha, \operatorname{PI} 3 \mathrm{~K} \beta, \mathrm{PI} 3 \mathrm{~K} \gamma$ and $\mathrm{PI} 3 \mathrm{~K} \delta$, is a competitive pan-PI3K inhibitor of ATP used in adult patients with recurrent follicular lymphoma, who have received at least two systematic treatments. Copanlisib has an obvious inhibitory activity against two PI3K $\alpha$ and PI3K $\delta$ subtypes expressed in malignant B cells (30).

Pictilisib (GDC-0941). Pictilisib was developed by Piramed Limited and licensed to Genentech in 2005. It has been shown to have a significant inhibitory effect on PI3K $\alpha$ and PI3K $\delta$ and a moderate selectivity for p1 $10 \beta$ and p110 $\gamma$ (31). At a dose of $150 \mathrm{mg} / \mathrm{kg}$, oral pictilisib had an inhibitory effect of $>80 \%$ on the growth of glioma and ovarian tumors in animals (32). Pictilisib has been shown to have minor side effects in previous clinical studies, and has been used in phase II clinical trials of breast cancer and non-small cell lung cancer (NSCLC) (33).

Buparlisib (BKM120). Buparlisib was developed by Novartis International $\mathrm{AG}$, and it targets $\mathrm{PI} 3 \mathrm{~K} \alpha, \mathrm{PI} 3 \mathrm{~K} \beta, \mathrm{PI} 3 \mathrm{~K} \gamma$ and $\mathrm{PI} 3 \mathrm{~K} \delta$ for the treatment of metastatic estrogen receptor $(\mathrm{ER})^{+} /$human epidermal growth factor receptor 2 (HER2) breast cancer (34). Novartis is also conducting phase II clinical studies for the treatment of follicular lymphoma, gastrointestinal stromal tumors, mantle cell lymphoma, prostate cancer, diffuse large B-cell lymphoma, ovarian cancer, NSCLC, hepatocellular carcinoma, bone marrow fibrosis, advanced endometrial cancer, melanoma, bladder, pancreatic cancer and malignant gliomas (35-38).

Taselisib (GDC-0032). Taselisib was developed by Genentech, Inc., and is an effective PI3K inhibitor. It targets mutant PI3KCA and can inhibit the activity of PI3K $\alpha, \operatorname{PI} 3 K \beta$ and PI3K $\gamma(39,40)$. At present, phase III clinical studies for the treatment of $\mathrm{ER}^{+}$breast cancer in postmenopausal women and NSCLC are underway.

Pilaralisib (XL147, SAR245408). Pilaralisib was developed by Exelixis, Inc., and Sanofi S.A. was granted the right to treat solid tumors in 2009. Pilaralisib is an oral biocompatible PI3K inhibitor that can inhibit PI3K $\alpha, \mathrm{PI} 3 \mathrm{~K} \beta, \mathrm{PI} 3 \mathrm{~K} \gamma$ and PI3Kס. It has been in a phase I clinical trial for the treatment of lymphoma and solid tumors (41-43).

ZSTK474. Jointly developed by Nippon Pharmaceutical Industry Co., Ltd. and the Japan Cancer Research Institute, ZSTK474 targets class I PI3K and has the most significant effect on PI3KS (44). Studies on the use of ZSTK474 for the treatment of advanced solid malignancies are in phase I clinical trials (45).

Sonolisib (PX-866). Developed by Oncothyreon Inc., sonolisib can covalently bind to Lys802 residues in the ATP binding site of p110 kinase catalytic subunit and irreversibly inhibit class I PI3K. It exerts an inhibitory activity against $\mathrm{PI} 3 \mathrm{~K} \alpha, \mathrm{PI} 3 \mathrm{~K} \beta$, $\mathrm{PI} 3 \mathrm{~K} \gamma$ and PI3Kס. Sonolisib is mainly used in the treatment of pleomorphic glioblastoma and metastatic prostate cancer, and has been entered into phase II clinical trials $(46,47)$. 
AMG511. An orally effective class I pan-PI3K inhibitor, AMG511 has a strong inhibitory effect on PI3K $\alpha, \operatorname{PI} 3 \mathrm{~K} \beta$, $\mathrm{PI} 3 \mathrm{~K} \gamma$ and $\mathrm{PI} 3 \mathrm{~K} \delta$, and can produce a strong anticancer effect in many tumor cell lines by inducing $\mathrm{G}_{1}$ phase arrest. In xenografts with PTEN deletion, KRAS mutation and HER2 amplification, AMG511 can significantly inhibit tumor growth (48).

PA799. Developed by Chugai Pharmaceutical Co., Ltd. and later granted the right of early oncology research to Menarini Group, PA799 selectively inhibits class I PI3K (PI3K $\alpha$, $\mathrm{PI} 3 \mathrm{~K} \beta, \mathrm{PI} 3 \mathrm{~K} \gamma$ and PI3K $\delta$ ), but has a lower inhibitory effect on class II and III PI3K. PA799 has a stronger inhibitory effect on tumors with PIK3CA mutations compared with tumors with wild type PI3KCA, and can reduce the phosphorylation level of p70S6K, 4E-BP1 and other factors downstream of AKT (49).

\section{Biochemical properties of buparlisib}

Buparlisib is a highly efficient and selective pan-class I PI3K inhibitor that belongs to the family of 2,6-dimorpholinopyrimidine-derived compounds (34). It selectively inhibits four isomers of PI3K, PI3K $\alpha$, PI3K $\beta$, PI3K $\gamma$ and PI3K 8 , by competitively binding to the lipid kinase domain on ATP, and it has an important role in inihibiting proliferation, promoting apoptosis and inihibiting angiogenesis, by antagonizing the PI3K/AKT pathway $(50,51)$. Due to the similarity in their catalytic domain, buparlisib is considered to also inhibit mTOR.

In clinical studies, buparlisib is often used in combination with other drugs or methods, the most common being synergistic treatment with the poly (ADP-ribose) polymerase (PARP) inhibitor olaparib in BRCA1-related triple negative breast cancer $(52,53)$. The combined use of drugs is prone to serious side effects. In order to avoid this issue and also ensure the therapeutic effect, researchers found through the detailed analysis of the chemical structure of buparlisib that, in addition to the binding region of ATP, there is also an idle morpholine group in buparlisib; therefore, a connector could be used to replace it and combine it with PARP inhibitors (54).

\section{Pharmacokinetic characteristics of buparlisib}

In a variety of animal models, it has been found that buparlisib has a fast absorption rate, high bioavailability and excellent pharmacokinetic characteristics (50). Following the oral administration of buparlisib at a dose of $60 \mathrm{mg} / \mathrm{kg}$, the peak of pharmacokinetics was reached in tumor tissue and plasma within $1 \mathrm{~h}$, and the phosphorylation of AKT was fully inhibited (55). This phase I clinical study also demonsstrated that buparlisib was rapidly absorbed following oral administration, and reached the peak concentration within $1.5 \mathrm{~h}$ (55). Next, the plasma concentration of buparlisib decreased doubly exponentially. In addition, buparlisib has been reported to efficiently break through the blood-brain barrier $(56,57)$. A phase I/II study showed that the combined use of buparlisib and trastuzumab did not affect the pharmacokinetic characteristics of buparlisib (58).

\section{Preclinical research on buparlisib}

Inducing apoptosis, blocking cell cycle progression and promoting cell death is one of the key goals in the treatment of the majority of tumors. Buparlisib has been proven to have an effect in apoptosis and cell cycle in breast, ovarian and intestinal cancer, glioma, lung cancer, head and neck squamous cell carcinoma (HNSCC) and several other cancer types. In addition, buparlisib has also been closely associated with oxidative phosphorylation, inflammation, cytoskeleton and other biological processes. The in-depth study of buparlisib has also provided data on its combination with other drugs for the treatment of a variety of cancer types, providing more directions for the clinical treatment of cancer.

Buparlisib induces apoptosis and inhibits proliferation. Bavelloni et al (59) showed that inhibition of the cell cycle induced by buparlisib in osteosarcoma varied slightly depending on the cell line. For example, HOS cells were arrested at the $\mathrm{G}_{2} / \mathrm{M}$ phase, $\mathrm{U} 2 \mathrm{OS}$ cells were arrested at the $\mathrm{G}_{1}$ phase, while MG-63 cells were arrested at the sub- $G_{1}$ phase, following buparlisib treatment (59). The buparlisib-induced apoptosis of osteosarcoma cells was characterized by an increase in endogenous caspase-3 activity (59). In addition, a specific apoptosis-promoting mechanism that inhibits the triggering of PI3K in lymphoid carcinoma was reported by Müller et al (60). This study showed that buparlisib could change the conformation of Bax and Bcl-2 homologous antagonist killer (Bak), lead to $\mathrm{N}$-terminal exposure and protein activation, and then affect the transcription of p53 upregulated modulator of apoptosis and Harakiri, Bcl-2 interacting protein, accompanied by the destruction of mitochondrial membrane potential and mitosis block; this indicated that buparlisib induced apoptosis in mitochondria (60). Buparlisib has also been shown to induce apoptosis in neuroblastoma, and the induction of apoptosis depends on the caspase-mediated pathway, which is associated with caspase 3/7 and cleaved-PARP (61). A previous study has shown that buparlisib can lead to cell cycle arrest and apoptosis in the $\mathrm{G}_{2} / \mathrm{M}$ phase of T-cell acute lymphoblastic leukaemia (TALL) cells and T lymphoblasts in patients, and that changes in the expression of cleaved-caspases $2 / 3 / 9$ occur in a time and concentration-dependent manner (62). Even when co-cultured with MS-5 stromal cells simulating the bone marrow microenvironment, buparlisib was shown to maintaine its proapoptotic activity against Jurkat cells. In addition, buparlisib has been shown to have a synergistic effect with chemotherapeutic drugs currently used to treat patients with TALL without any toxicity (62). In ovarian cancer, the combined inhibition of SH2 containing protein tyrosine phosphatase-2 and PI3K synergistically inhibited cell proliferation and survival, partly by activating proapoptotic $\mathrm{Bcl}-2$ interacting mediator of cell death and inhibiting c-Myc (63). Buparlisib is most widely used in breast cancer and HNSCC. In addition to affecting the growth and cell cycle arrest of breast cancer cells $(64,65)$, buparlisib can also be combined with the inhibition of the c-Myc-mediated human telomerase reverse transcriptase (hTERT) to enhance the arsenic trioxide (ATO)-induced anti-proliferative effect (66). In a study on colorectal cancer, Solberg et al (67) found that buparlisib can block HCT-15 cells at the $\mathrm{G}_{1}$ phase, accompanied by a decrease in c-Myc and 
cyclin D1 and increase in cyclin-dependent kinase inhibitor (CDKN) 1A and CDKN1B. Buparlisib can block the cell cycle of A549 and H522 lung cancer cells at the $\mathrm{G}_{1} / \mathrm{S}$ and $\mathrm{G}_{2}$ phase, respectively (68). Compared with another pan-PI3K inhibitor PX-866 $\left(\mathrm{G}_{0} / \mathrm{G}_{1}\right)$, the cell cycle arrest effect of buparlisib on Jurkat cells mainly occurred at the $\mathrm{G}_{2} / \mathrm{M}$ phase, and the apoptosis induced by buparlisib was more significant (69). A previous study has shown that buparlisib is involved in two main pathways of cell death in leukemic cells: Death receptor and mitochondrial mediation (70). The increase of FAS and the cleavage of procaspase- 8 and procaspase- 3 suggest that the exogenous pathway of apoptosis is activated. At the same time, it was observed that, following buparlisib treatment, the change in cleaved-procaspase-9, decrease in Bad phosphorylation and upregulation of $\mathrm{Bax} / \mathrm{Bcl}-2$ ratio were all associated with mitochondrial apoptosis (71). The regulation of cleaved-caspase-3 by buparlisib was also observed in the Cal27 and Scc 25 oral squamous cell carcinoma cells lines (72).

In the aforementioned studies, buparlisib directly inhibited the proliferation or apoptosis pathway to exert its pharmacological effect. Recent studies have indicated that buparlisib can also affect other targets. Certain studies have shown that PTEN may be the key to determining the therapeutic effect of buparlisib. In endometrial carcinoma, PTEN deletion/mutation has been shown to increase the effect of buparlisib plus PARP inhibitors in vivo and in vitro $(73,74)$. Mutation of p53 is closely associated with the apoptosis of tumor cells. When evaluating the exogenous apoptosis pathway, Bashash et al (75) found that the inhibition of PI3K could increase the positive percentage of intracellular Annexin-V/PI and the reactive oxygen species (ROS) levels in p53 mutant NB4 and p53 wild-type NALM-6 cells in a concentration-dependent manner. Buparlisib could inhibit the expression of the NF- $\mathrm{NB}$ target gene in NB4 cells, in which the expression of $\mathrm{p}-\mathrm{I} \kappa \mathrm{B}$ was downregulated in a concentration-dependent manner, and inhibit the transcriptional activity of the apoptosis target gene. In NALM-6 cells, buparlisib lead to changes in the transcriptional activities of $\mathrm{p} 73$ and FOXO3a, which may be associated with ROS-mediated apoptosis (75).

As a member of the $\mathrm{NAD}^{+}$-dependent class III histone deacetylase family, sirtuin (SIRT) 1 acts as an important negative regulator of p53 and an anti-apoptotic element (76). Of note, later studies by Bashash et al $(75,77)$ have shown that buparlisib can reduce the transcriptional level of hTERT in NB4 cells, significantly enhance the effect of ATO on cells, reduce the expression of SIRT1, expand the effect of changes in factors such as MCL1, X-linked inhibitor of apoptosis, cellular inhibitor of apoptosis protein 1, Bcl-2, Bax and Bad on apoptosis, and induce $\mathrm{G}_{1}$-phase arrest (77). Certain studies have shown that the sensitivity of leukemic cells to isomer-specific PI3K inhibitors has nothing to do with the mutation/inactivation of PTEN. Similarly, in myeloma, it was found by Safaroghli-azar et al (78) that there was no significant correlation between the effect of buparlisib and the state of PTEN in cells. Buparlisib can upregulate the expression of SIRT1 in cells, inhibit the activity of NF- $\kappa \mathrm{B}$ and block the $\mathrm{G}_{2} / \mathrm{M}$ phase of the cell cycle (78). SIRT6, the downstream target of FoxO3a, is also one of the new targets of buparlisib. SIRT6 can activate Bax and the mitochondrial pathway to promote cell apoptosis. It was reported by Zhang et al (79) that buparlisib can reduce the levels of phosphorylated FOXO3a and increase the expression of SIRT6 in colorectal cancer cells and patient tissue samples; the possible mechanism underlying this effect appears to be that buparlisib can dephosphorylate FoxO3a and increase the levels of FoxO3a on the SIRT6 promoter, triggering SIRT6 transcription. Activated SIRT6 acetylates the histone H3K9 site of survivin and other factors, downregulates its transcription and finally initiates mitochondrial apoptosis (79).

Certain studies have shown that the anticancer effect of buparlisib is associated with the PIK3CA mutation status. In a xenograft model of bone metastasis established using PIK3CA-mutated NCI-H460-luc2 cells, buparlisib could induce apoptosis in tumor tissues (80). Of note, with the exception of the upregulation of caspase- 3 activity, no change in the expression levels of other pro or antiapoptotic proteins, such as Bcl-2 and Bax, was observed (80). It has been suggested that buparlisib may affect the occurrence of apoptosis through other mechanisms. It is worth noting that the study by Trautmann et al (81) in myxoid liposarcoma demonstrated that the effect of buparlisib on apoptosis was more significant in cells with a PIK3CA-H1047R mutation.

Combination therapy of buparlisib. A study on colorectal cancer showed that buparlisib could significantly increase the expression of p53 in HCT-15 cells, and did not induce the apoptosis phenotype in COLO320DM cells (67). By contrast, when combined with the tankyrase inhibitor G007-LK, buparlisib significantly induced apoptosis, regardless of the nuclear catenin and FOXO3a levels (67). Buparlisib alone blocked the $G_{1} / S$ phase of A549 cells, but its combination with MEK162 (MEK1/2 inhibitor) was more effective in inducing $\mathrm{G}_{1}$ phase arrest (68). In a study on biliary tract cancer, it was found that buparlisib buparlisib did not inhibit cell proliferation, migration and cell cycle progression in cells harbouring PI3KCA/KRAS mutations. However, when combined with MEK162, the resistance of PI3KCA/KRAS-mutant cells to buparlisib was overcome (82). Prima-1Met is a low molecular weight compound that can restore the transcriptional function of mutant p53 (83). Buparlisib and Prima-1Met can serve a combined role in thyroid cancer cells, and the possible mechanisms include the activation of the caspase apoptosis pathway and the inhibition of the PI3K/AKT/mTOR and cleavage and polyadenylation specific factor 4/hTERT pathways (84). Aasen et al (85) studied the role and mechanism of buparlisib using BRAF-mutant cells extracted from patients with melanoma brain metastases. Following the use of buparlisib alone, the expression of cytochrome C,TRAIL-R2 and Fas increased, while that of heat shock protein 27 decreased. In combination with the MEK1/2 inhibitor trametinib, the expression of apoptosis proteins Bad, caspase-3, p27/kip1 and TNFR1 was significantly increased, and that of apoptosis inhibitors such as Bcl-2, cellular inhibitor of apoptosis protein 1, claspin, hypoxia-inducible factor $1-\alpha, 70-\mathrm{kDa}$ heat shock protein and survivin was significantly downregulated following single or combined treatment (85).

Tumor cell cycle arrest and apoptosis induced by buparlisib have also been demonstrated in gliomas and undifferentiated thyroid carcinomas. Buparlisib increased the expression of cleaved-caspase-3 and Bax in C6 glioma cells treated with 
the chemotherapy agent temozolomide (TMZ) (86). In vitro, apoptosis could be induced by the inhibition of PI3K and the use of volasertib, an experimental small molecule inhibitor of the polo-like kinase 1 protein (87). Of note, it was found by Sai et al (88) that buparlisib had an effect on the immune system in vivo, through orthotopic and allogeneic breast tumor transplantation models. Sai et al (88) showed that buparlisib effectively abrogated lung metastasis of breast cancer cells, as well as significantly altered the composition of tumor-infiltrating leukocytes at the primary or metastatic tumor site. By increasing the number of antitumor leukocytes in the tumor site, buparlisib could change the microenvironment and further hinder the growth of tumor cells (88). For that reason, buparlisib and the checkpoint inhibitor blocker anti-PD1 antibody may have potential as a combined therapy. Of note, buparlisib did not significantly reduce the cytotoxicity of T cells to target 4T1 tumor cells (88).

Although several studies have reported that buparlisib can induce apoptosis, Anisuzzaman et al (89) found that buparlisib alone did not induce apoptosis in HNSCC cells, but when combined with erlotinib it inhibited the Bcl-2 protein through 4E-BP1, thus connecting the mTOR protein translation and the intrinsic apoptotic pathways (89). Notably, other studies have also shown a contrary role for buparlisib alone in HNSCC cells. Yun et al (90) reported that buparlisib treatment upregulated interleukin (IL-6) levels and then activated ERK and STAT3 to promote the growth of HNSCC cells. Blocking the autocrine IL- 6 signaling pathway with small interfering RNA or neutralizing antibody for IL-6 receptor completely eliminated the buparlisib-induced activation of ERK and STAT3 and expression of c-Myc (90).

The combined use of PI3K and bromodomain and extraterminal domain protein (BET) inhibitors has also been reported. The combination of buparlisib and JQ1 synergistically inhibited the EGFR and c-Myc pathways in SACC-83 salivary gland adenoid cystic carcinoma cells and promoted the occurrence of apoptosis (91). Sakakibara et al (92) found that buparlisib strongly induced apoptosis and inhibited cell proliferation in individual myeloid/lymphoblastic leukemia cell lines resistant to binimetinib, a MEK1/2 inhibitor. It is worth noting that there was a higher level of phosphorylated AKT in binimetinib-resistant strains (92).

Finally, the most-well known mechanism for combination treatment of buparlisib is for PARP inhibitors. Certain studies have shown that buparlisib combined with PARP inhibitors can increase the $\gamma-\mathrm{H} 2 \mathrm{~A}$.X variant histone in various types of cancer, decrease the expression of homologous recombinant (HR) repair protein RAD51 and destroy the HR repair ability of cells $(73,74,93)$. Another study demonstrted that the lack of AT-rich interaction domain 1A (ARID1A) expression renders gastric cancer cells sensitive to PI3K and PARP inhibitors (94).

Other functions. Several studies have revealed a variety of additional anticancer effects of buparlisib. Speranza et al (50) demonstrated for the first time that buparlisib may be associated with the cytoskeleton. Speranza et al (50) have shown that buparlisib significantly inhibited the adhesion of G9-copGFP cells, indicating that it can affect the ability of cells to form firm contact. Following treatment of U251 cells with buparlisib for $24 \mathrm{~h}$, the cells became round, which indicated that buparlisib may destroy the microtubule cytoskeleton. It is known that there are tumor-inititating cell (TIC) subsets in cancer with an ability for self-renewal, very similar to that of embryonic stem cells (95). These TIC subsets can regenerate themselves and other cancer cells in appropriate microenvironmental niches (95). Cells that express both aldehyde dehydrogenases $(\mathrm{ALDH})^{+}$and cluster of differentiation (CD) $44^{+} / \mathrm{CD} 24$ may have the strongest carcinogenesis and self-renewal abilities (95). A previous study has shown that buparlisib can selectively target $\mathrm{CD}_{4} 4^{+} / \mathrm{CD} 24^{-}$in $\mathrm{ALDH}^{+} \mathrm{MDA}-\mathrm{MB} 231$ cell subsets (96). Buparlisib can also affect non-homologous end joining (NHEJ), an error-prone repair mechanism that leads to the accumulation of damaged DNA, which in turn leads to cell death. Zhao et al (97) showed that, although buparlisib had little effect on the cell cycle of CAL51 and MDA-MB-231 cells, it promoted the repair of NHEJ and inhibited the growth of breast cancer cell lines. Buparlisib can also exert its antitumor effects by inhibiting the expression of golgi membrane protein 1 (GOLM1) in prostate cancer cells (98). GOLM1 has been identified as a new biomarker for the diagnosis of prostate cancer (99). Compared with control cells, buparlisib treatment attenuated the apoptosis inhibition of DU145 cells caused by the overexpression of GOLM1 (98). In gliomas, buparlisib upregulated the expression of TRAIL-R2, thus promoting the TRAIL-induced apoptosis in gliomas, but does not upregulate the expression of TRAIL-R1, another major TRAIL-associated death receptor (100).

\section{Ongoing clinical trials on buparlisib}

Several clinical trials are currently evaluating buparlisib alone or as part of combination therapy in patients with cancer. Phase III trial NCT04338399 plans to recruit 483 adult patients with recurrent or metastatic HNSCC who failed to receive cisplatin or anti-PD1-based treatment. The survival of patients treated with paclitaxel alone or in combination with buparlisib will be examined. The completion time of the study is tentatively scheduled for December 2023. NCT01551030 is an ongoing pharmacodynamic study on buparlisib in patients with metastatic urothelial cancer (phase II clinical trial). The aim of the first part of the trial is to examine whether drugs can reduce or slow down cancer growth in patients. The aim of the second part is to explore whether buparlisib can reduce or slow down the growth of urothelial tumors in patients with certain genetic mutations that cause such tumors. NCT02756247 is an ongoing Phase Ib clinical trial. The purpose of the study is to examine whether the combination of buparlisib and ibrutinib can achieve a superior therapeutic effect in patients with recurrent or refractory follicular lymphoma, mantle cell lymphoma or diffuse large B-cell lymphoma. The NCT02113878 trial, which is being conducted in patients with locally advanced HNSCC, is also in phase Ib; its aim is to determine the maximum dose of buparlisib/cisplatin that can be combined with radiotherapy and to evaluate the tolerance of this combination in high-risk patients with locally advanced HNSCC.

A phase I clinical trial (NCT01623349) of recurrent triple-negative breast cancer and high-grade serous ovarian cancer is underway to determine the safety of drug combinations. The aim of that study is to determine the highest dose that can be safely administered, and to explore whether a 
combination of buparlisib/BYL719 and olaparib is effective for both cancer types. It is worth noting that the phase II NCT02220855 trial showed that patients with recurrent or refractory thymoma took oral buparlisib for a median of 11.1 months, and $>70 \%$ of the patients developed fatigue and anorexia. In addition, $>60 \%$ of patients developed skin and subcutaneous tissue-related diseases, such as itching, rashes and acne. Unfortunately, due to a lack of funding, the study originally planned to recruit 16 patients, but only 14 patients were actually recruited (101). NCT02049541, a phase I clinical trial, studied the side effects and optimal dose of buparlisib in conjunction with rituximab for the treatment of patients with recurrent or refractory low-grade B-cell lymphoma, and evaluated the efficacy of the combination of the two drugs. Finally, the multicenter, open label phase II clinical trial NCT02159066 is evaluating the antitumor activity of advanced LGX818/MEK162 therapy in combination with targeted drugs (buparlisib, LEE011, BGJ398 or INC280) in advanced melanoma with BRAF mutations, as well as the safety and tolerance of the new triple combination. A summary of ongoing clinical trials involving buparlisib is shown in Table I.

\section{Conclusion}

In recent years, increasing attention has been paid to tumor-targeted therapies. The clinical benefit of buparlisib has been demonstrated in patients with solid tumors and hematological malignancies. A global clinical phase II trial using paclitaxel in HNSCC has been completed with a manageable safety profile. Buparlisib currently has fast-track status with the FDA. In addition, it has been shown to have a good brain permeability, independent of the blood-brain barrier efflux transporter (57). These characteristics make buparlisib an ideal option for intracranial targeted therapeutic strategies involving PI3K inhibition. Buparlisib in combination with TMZ has been shown to enhance the ability of TMZ to induce apoptosis $(86,87)$. Therefore, it can be hypothesized that it is more likely to be used in combination with existing chemotherapeutic agents in cases where the efficacy of buparlisib is not satisfactory due to tumor resistance, a hypothesis that can provide new directions for the clinical therapy of glioma.

In basic research studies, the main function of buparlisib is to induce an apoptotic phenotype in tumor cells (Table II). Mechanistically, buparlisib has been shown to affect various apoptosis-related factors, including caspases, NF- $\kappa \mathrm{B}$, Fas, FOX3a and ROS. The effects on the cell cycle also ranged from arrest in the sub- $G_{1}$ to the $G_{2} / M$ phases, which appeared to depend on the cancer type and cellular background. A previous study on the chemical structure of buparlisib has indicated that buparlisib interacts with Tyr55, the catalytic residue of Aldo-keto reductase family 1 member C3 (AKR1C3), through trifluoromethyl (102), an aldehyde and ketone reductase widely distributed in human tissues (103). AKR1C3 is closely associated with several types of cancer and is involved in several metabolic pathways, implying that buparlisib may serve a potential role in metabolic regulation.

Jiang et al (104) found that EGFR/KRAS mutations do not appear to affect the sensitivity of cancer cells to buparlisib treatment. Buparlisib can induce apoptosis and increased caspase-3 activity in PIK3CA-mutated NCI-H460 lung cancer

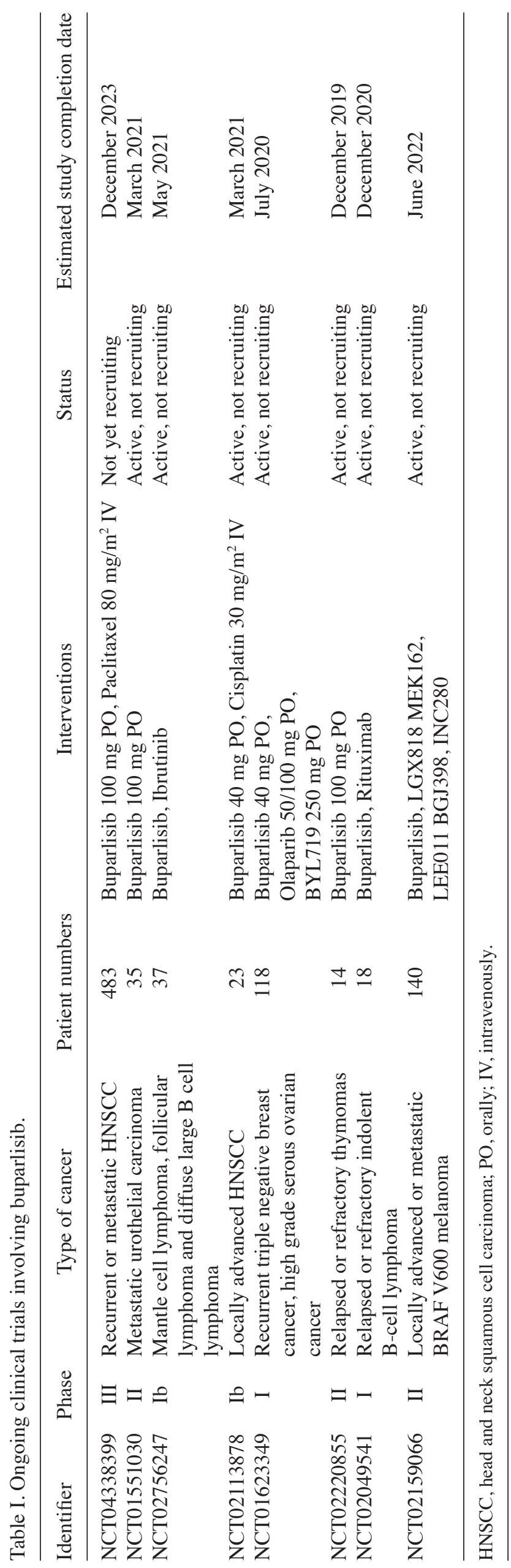


Table II. List of studies demonstrating the effect of buparlisib in cancer cell apoptosis.

\begin{tabular}{|c|c|c|c|}
\hline First author, year & Type of cancer & Main factors ${ }^{\mathrm{a}}$ & (Refs.) \\
\hline Bavelloni et al, 2019 & Osteosarcoma & Endogenous cas3 & $(59)$ \\
\hline Müller et al, 2018 & B-cell non-Hodgkin lymphoma & Bax/Bak, Puma, Hrk & $(60)$ \\
\hline Zhao et al, 2017 & Medulloblastoma & Cas3/7, Clv-cas3, Clv-PARP & $(61)$ \\
\hline Lonetti et al, 2014 & Acute lymphoblastic leukaemia & Clv-cas $2 / 3 / 9$ & $(62)$ \\
\hline Sun et al, 2019 & Ovarian cancer & Bim, Bad & $(63)$ \\
\hline Pereira et al, 2015 & Acute lymphoblastic leukaemia & Clv-procas3/8/9, Fas, Bad, Bax/Bcl-2 & $(71)$ \\
\hline Liu et al, 2019 & Oral squamous cell carcinoma & Clv-cas3 & $(72)$ \\
\hline Bashash et al, 2016 & Acute leukaemia & $\mathrm{Bax}, \mathrm{Bad}$ & $(75)$ \\
\hline Bashash et al, 2018 & Acute promyelocytic leukaemia & $\begin{array}{l}\text { Bax, Bad, Bcl-2, survivin, MCL1, } \\
\text { XIAP, c-IAP1 }\end{array}$ & $(77)$ \\
\hline Safaroghli-Azar et al, 2019 & Multiple myeloma & $\begin{array}{l}\text { Cas3, MCL1, Bcl-2, c-IAP1, c-IAP2, } \\
\text { survivin }\end{array}$ & $(78)$ \\
\hline Zhang et al, 2019 & Colon cancer & Cyto-c, Clv-cas3/9, survivin & $(79)$ \\
\hline Wang et al, 2019 & Non-small cell lung cancer & Cas3 & $(80)$ \\
\hline Trautmann et al, 2019 & Myxoid liposarcoma & Clv-PARP, cas3/7 & $(81)$ \\
\hline Li et al, 2018 & Thyroid cancer & Cyto-c & $(84)$ \\
\hline Aasen et al, 2019 & Melanoma & Clv-cas3, c-IAP1, Clv-P, XIAP, survivin, Fas & $(85)$ \\
\hline Li et al, 2017 & Glioblastoma & Clv-cas3, Bax & $(86)$ \\
\hline Anisuzzaman et al, 2017 & $\begin{array}{l}\text { Head and neck squamous cell } \\
\text { carcinoma }\end{array}$ & Bcl-2, Bcl-xL, MCL1 & $(89)$ \\
\hline Liu et al, 2019 & Adenoid cystic carcinoma & Clv-cas3 & $(91)$ \\
\hline Sakakibara et al, 2019 & Acute myeloid leukaemia & Clv-P, Bcl-2, Bcl-xL, Bcl-Xs & $(92)$ \\
\hline Bian et al, 2018 & Endometrial cancer & Clv-PARP, Clv-cas3 & $(73)$ \\
\hline Wang et al, 2016 & Ovarian cancer & Clv-PARP, Clv-cas3 & $(93)$ \\
\hline Yang et al, 2018 & Gastric cancer & Clv-PARP & (94) \\
\hline Zhao et al, 2018 & Triple-negative breast cancer & Clv-PARP & $(97)$ \\
\hline Foster et al, 2015 & Malignant glioma & $\begin{array}{l}\text { Clv-PARP, Clv-cas3/7/8/9, Bcl-2, } \\
\text { Bcl-xL, MCL1 }\end{array}$ & $(100)$ \\
\hline
\end{tabular}

${ }^{a}$ Main factors, the apoptotic factors that were directly affected by buparlisib. Puma, p53 upregulated modulator of apoptosis; Hrk, harakiri BCL2 interacting protein; Cas, caspase; Clv, cleaved; PARP, poly (ADP-ribose) polymerase; procas, procaspase; XIAP, X-linked inhibitor of apoptosis; c-IAP, cellular inhibitor of apoptosis protein; Cyto-c, cytochrome-c.

cells, but the expression levels of the apoptosis-related proteins Bcl-2 and Bax were unaffected (80); this suggests that buparlisib may affect the occurrence of apoptosis through other mechanisms. In combination, these findings indicate that the mechanism of buparlisib-induced apoptosis in different tumor cells has not been fully elucidated, therefore further studies will be required to uncover more targets and clinical applications of buparlisib.

Of note, the most common side effect of buparlisib described in early clinical trials is hyperglycemia, which is a common adverse reaction to PI3K inhibitors (55). Later trials have shown that buparlisib also appears to have effects on the respiratory, digestive and immune systems (NCT01385293, NCT01487265). Therefore, the immunosuppressive characteristics of buparlisib should be taken into account when using it in basic or clinical studies in the future, to minimize its adverse effects on immune function. It has been shown that buparlisib impairs tumor cell growth by regulating leukocytes. By contrast, buparlisib was not found to significantly reduce the cytotoxic effect of T cells on 4T1 tumor cells (88). This suggests that the effects of buparlisib on the human immune system are complex and diverse, and additional evidence on the combination of buparlisib and immune drugs is required.

In conclusion, the potential beneficial role of buparlisib in the therapy of various types of cancer has been increasingly recognized, but the uderlying mechanisms remain to be fully elucidated. The present review aimed to summarize the current knowledge on buparlisib, highlighting that buparlisib has the potential to serve as an effective, novel and efficient cancer treatment. 


\section{Acknowledgements}

Not applicable.

\section{Funding}

The present review was supported by the research start-up fund of Southwest Medical University (grant no. 00040173).

\section{Availability of data and materials}

Not applicable.

\section{Authors' contributions}

JX and JY conceived the review and wrote the manuscript. YG and JY collected the relevant literature and revised the manuscript. All authors read and approved the final manuscript.

\section{Ethics approval and consent to participate}

Not applicable.

\section{Patient consent for publication}

Not applicable.

\section{Competing interests}

The authors declare that they have no competing interests.

\section{References}

1. Fruman DA, Meyers RE and Cantley LC: Phosphoinositide kinases. Annu Rev Biochem 67: 481-507, 1998.

2. Katso R, Okkenhaug K, Ahmadi K, White S, Timms J and Waterfield MD: Cellular function of phosphoinositide 3-kinases: Implications for development, homeostasis, and cancer. Annu Rev Cell Dev Biol 17: 615-675, 2001.

3. Nobukuni T, Kozma SC and Thomas G: hvps34, an ancient player, enters a growing game: mTOR Complex1/S6K1 signaling. Curr Opin Cell Biol 19: 135-141, 2007.

4. Dornan GL and Burke JE: Molecular mechanisms of human disease mediated by oncogenic and primary immunodeficiency mutations in Class IA phosphoinositide 3-kinases. Front Immunol 9: 575, 2018

5. Bader AG, Kang S, Zhao L and Vogt PK: Oncogenic PI3K deregulates transcription and translation. Nat Rev Cancer 5: 921-929, 2005.

6. Suire S, Coadwell J, Ferguson GJ, Davidson K, Hawkins P and Stephens L: p84, a new Gbetagamma-activated regulatory subunit of the type IB phosphoinositide 3-kinase p110gamma. Curr Biol 15: 566-570, 2005.

7. Voigt P, Dorner MB and Schaefer M: Characterization of p87PIKAP, a novel regulatory subunit of phosphoinositide 3-kinase gamma that is highly expressed in heart and interacts with PDE3B. J Biol Chem 281: 9977-9986, 2006.

8. Gulluni F, De Santis MC, Margaria JP, Martini M and Hirsch E: Class II PI3K functions in cell biology and disease. Trends Cell Biol 29: 339-359, 2019.

9. Rommel C: Taking PI3Kס and PI3K $\gamma$ one step ahead: Dual active $\mathrm{PI} 3 \mathrm{~K} \delta / \gamma$ inhibitors for the treatment of immune-mediated inflammatory diseases. Curr Top Microbiol Immunol 346 279-299, 2010.

10. Mayer IA and Arteaga CL: PIK3CA activating mutations: A discordant role in early versus advanced hormone-dependent estrogen receptor-positive breast cancer? J Clin Oncol 32: 2932-2934, 2014.
11. Martínez-Sáez O, Chic N, Pascual T, Adamo B, Vidal M, González-Farré B, Sanfeliu E, Schettini F, Conte B, Brasó-Maristany F, et al: Frequency and spectrum of PIK3CA somatic mutations in breast cancer. Breast Cancer Res 22: 45, 2020.

12. Stephens LR, Jackson TR and Hawkins PT: Agonist-stimulated synthesis of phosphatidylinositol $(3,4,5)$-trisphosphate: A new intracellular signalling system? Biochim Biophys Acta 1179: 27-75, 1993.

13. Vanhaesebroeck B, Leevers SJ, Ahmadi K, Timms J, Katso R, Driscoll PC, Woscholski R, Parker PJ and Waterfield MD: Synthesis and function of 3-phosphorylated inositol lipids. Annu Rev Biochem 70: 535-602, 2001.

14. Foster FM, Traer CJ, Abraham SM and Fry MJ: The phosphoinositide (PI) 3-kinase family. J Cell Sci 116: 3037-3040, 2003.

15. Wymann MP, Zvelebil M and Laffargue M: Phosphoinositide 3-kinase signalling-which way to target? Trends Pharmacol Sci 24: 366-376, 2003

16. Oudit GY, Sun H, Kerfant BG, Crackower MA, Penninger JM and Backx PH: The role of phosphoinositide-3 kinase and PTEN in cardiovascular physiology and disease. J Mol Cell Cardiol 37: 449-471, 2004.

17. Maehama T, Taylor GS and Dixon JE: PTEN and myotubularin: Novel phosphoinositide phosphatases. Annu Rev Biochem 70: 247-279, 2001

18. DiNitto JP, Cronin TC and Lambright DG: Membrane recognition and targeting by lipid-binding domains. Sci STKE 2003: re16, 2003

19. Miralem T, Lerner-Marmarosh N, Gibbs PE, Jenkins JL, Heimiller $\mathrm{C}$ and Maines MD: Interaction of human biliverdin reductase with Akt/protein kinase $\mathrm{B}$ and phosphatidylinositol-dependent kinase 1 regulates glycogen synthase kinase 3 activity: A novel mechanism of Akt activation. FASEB J 30: 2926-2944, 2016.

20. Kang SA, O'Neill DJ, Machl AW, Lumpkin CJ, Galda SN, Sengupta S, Mahoney SJ, Howell JJ, Molz L, Hahm S, et al: Discovery of small-molecule selective mTORC1 inhibitors via direct inhibition of glucose transporters. Cell Chem Biol 26: 1203-1213.e13, 2019.

21. Hua H, Kong Q, Zhang H, Wang J, Luo T and Jiang Y: Targeting mTOR for cancer therapy. J Hematol Oncol 12: 71, 2019.

22. Jacinto E, Loewith R, Schmidt A, Lin S, Rüegg MA, Hall A and Hall MN: Mammalian TOR complex 2 controls the actin cytoskeleton and is rapamycin insensitive. Nat Cell Biol 6: 1122-1128, 2004.

23. Jacinto E, Facchinetti V, Liu D, Soto N, Wei S, Jung SY, Huang Q, Qin J and Su B: SIN1/MIP1 maintains rictor-mTOR complex integrity and regulates Akt phosphorylation and substrate specificity. Cell 127: 125-137, 2006.

24. Engelman JA, Luo J and Cantley LC: The evolution of phosphatidylinositol 3-kinases as regulators of growth and metabolism. Nat Rev Genet 7: 606-619, 2006.

25. Darici S, Alkhaldi H, Horne G, Jørgensen HG, Marmiroli S and Huang X: Targeting PI3K/Akt/mTOR in AML: Rationale and clinical evidence. J Clin Med 9: 2934, 2020.

26. Raza W, Luqman S and Meena A: Prospects of tangeretin as a modulator of cancer targets/pathways. Pharmacol Res 161: $105202,2020$.

27. Wen W, Marcinkowski E, Luyimbazi D, Luu T, Xing Q, Yan J, Wang Y, Wu J, Guo Y, Tully D, et al: Eribulin synergistically increases anti-tumor activity of an mTOR inhibitor by inhibiting $\mathrm{pAKT} / \mathrm{pS} 6 \mathrm{~K} / \mathrm{pS} 6$ in triple negative breast cancer. Cells 8: 1010, 2019.

28. Meng C, Xia Q, Wu H, Huang H, Liu H, Li Y, Zhang F and Song W: Photobiomodulation with $630-\mathrm{nm}$ LED radiation inhibits the proliferation of human synoviocyte MH7A cells possibly via TRPV4/PI3K/AKT/mTOR signaling pathway. Lasers Med Sci 35: 1927-1936, 2020.

29. Verret B, Cortes J, Bachelot T, Andre F and Arnedos M: Efficacy of PI3K inhibitors in advanced breast cancer. Ann Oncol 30 (Suppl): 10:x12-x20, 2019.

30. Liu N, Rowley BR, Bull CO, Schneider C, Haegebarth A, Schatz CA, Fracasso PR, Wilkie DP, Hentemann M, Wilhelm SM, et al: BAY 80-6946 is a highly selective intravenous PI3K inhibitor with potent $\mathrm{p} 110 \alpha$ and $\mathrm{p} 110 \delta$ activities in tumor cell lines and xenograft models. Mol Cancer Ther 12: 2319-2330, 2013. 
31. O'Brien C, Wallin JJ, Sampath D, GuhaThakurta D, Savage H, Punnoose EA, Guan J, Berry L, Prior WW, Amler LC, et al: Predictive biomarkers of sensitivity to the phosphatidylinositol 3 kinase inhibitor GDC-0941 in breast cancer preclinical models. Clin Cancer Res 16: 3670-3683, 2010.

32. Sarker D, Ang JE, Baird R, Kristeleit R, Shah K, Moreno V, Clarke PA, Raynaud FI, Levy G, Ware JA, et al: First-in-human phase I study of pictilisib (GDC-0941), a potent pan-class phosphatidylinositol-3-kinase (PI3K) inhibitor, in patients with advanced solid tumors. Clin Cancer Res 21: 77-86, 2015.

33. Raynaud FI, Eccles SA, Patel S, Alix S, Box G, Chuckowree I, Folkes A, Gowan S, De Haven Brandon A, Di Stefano F, et al: Biological properties of potent inhibitors of class I phosphatidylinositide 3-kinases: from PI-103 through PI-540, PI-620 to the oral agent GDC-0941. Mol Cancer Ther 8: 1725-1738, 2009.

34. Ragon BK, Kantarjian H, Jabbour E, Ravandi F, Cortes J, Borthakur G, DeBose L, Zeng Z, Schneider H,Pemmaraju N, et al: Buparlisib, a PI3K inhibitor, demonstrates acceptable tolerability and preliminary activity in a phase I trial of patients with advanced leukemias. Am J Hematol 92: 7-11, 2017.

35. Heudel PE, Fabbro M, Roemer-Becuwe C, Kaminsky MC, Arnaud A, Joly F, Roche-Forestier S, Meunier J, Foa C, You B, et al: Phase II study of the PI3K inhibitor BKM120 in patients with advanced or recurrent endometrial carcinoma: A stratified type I-type II study from the GINECO group. $\mathrm{Br}$ J Cancer 116: 303-309, 2017.

36. Matulonis UA, Wulf GM, Barry WT, Birrer M, Westin SN, Farooq S, Bell-McGuinn KM, Obermayer E, Whalen C, Spagnoletti T, et al: Phase I dose escalation study of the PI3kinase pathway inhibitor BKM120 and the oral poly (ADP ribose) polymerase (PARP) inhibitor olaparib for the treatment of high-grade serous ovarian and breast cancer. Ann Oncol 28 : 512-518, 2017

37. Bedard PL, Tabernero J, Janku F, Wainberg ZA, Paz-Ares L, Vansteenkiste J, Van Cutsem E, Pérez-García J, Stathis A, Britten $\mathrm{CD}$, et al: A phase Ib dose-escalation study of the oral pan-PI3K inhibitor buparlisib (BKM120) in combination with the oral MEK1/2 inhibitor trametinib (GSK1120212) in patients with selected advanced solid tumors. Clin Cancer Res 21: 730-738, 2015

38. Maira SM, Pecchi S, Huang A, Burger M, Knapp M, Sterker D, Schnell C, Guthy D, Nagel T, Wiesmann M, et al: Identification and characterization of NVP-BKM120, an orally available pan-class I PI3-kinase inhibitor. Mol Cancer Ther 11: 317-328, 2012.

39. Tamura K, Kodaira M, Shimizu C, Yonemori K, Yunokawa M, Shimomura A, Kobayashi T, Nakano K, Tomomatsu J, Ito Y, et al: Phase I study of taselisib in Japanese patients with advanced solid tumors or hormone receptor-positive advanced breast cancer. Cancer Sci 109: 1592-1601, 2018.

40. Juric D, Krop I, Ramanathan RK, Wilson TR, Ware JA, Sanabria Bohorquez SM, Savage HM, Sampath D, Salphati L, Lin RS, et al: Phase I dose-escalation study of taselisib, an oral PI3K inhibitor, in patients with advanced solid tumors. Cancer Discov 7: 704-715, 2017.

41. Edelman G, Rodon J, Lager J, Castell C, Jiang J, Van Allen EM, Wagle N, Lindeman NI, Sholl LM and Shapiro GI: Phase I trial of a tablet formulation of pilaralisib, a Pan-class I PI3K inhibitor, in patients with advanced solid tumors. Oncologist 23: 401-e38, 2018.

42. Wheler J, Mutch D, Lager J, Castell C, Liu L, Jiang J and Traynor AM: Phase I dose-escalation study of pilaralisib (SAR245408, XL147) in combination with paclitaxel and carboplatin in patients with solid tumors. Oncologist 22: 377-e37, 2017.

43. Abramson V, Supko J, Ballinger T, Cleary JM, Hilton JF, Tolaney SM, Chau NG, Cho DC, Pearlberg J, Lager J, et al: Phase Ib study of safety and pharmacokinetics of the PI3K inhibitor SAR245408 with the HER3-neutralizing human antibody SAR256212 in patients with solid tumors. Clin Cancer Res 23 : 3520-3528, 2017

44. Wang Y, Liu J, Qiu Y, Jin M, Chen X, Fan G, Wang R and Kong D: ZSTK474, a specific class I phosphatidylinositol 3-kinase inhibitor, induces G1 arrest and autophagy in human breast cancer MCF-7 cells. Oncotarget 7: 19897-19909, 2016.

45. Lockhart AC, Olszanski AJ, Allgren RL, Yaguchi S, Cohen SJ, Hilton JF, Wang-Gillam A and Shapiro GI: Abstract B271: A first-in-human phase I study of ZSTK474, an oral pan-PI3K inhibitor, in patients with advanced solid malignancies. Mol Cancer Ther 12: B271-B271, 2013
46. Hotte SJ, Chi KN, Joshua AM, Tu D, Macfarlane RJ, Gregg RW, Ruether JD, Basappa NS, Finch D, Salim M, et al: A phase II study of PX-866 in patients with recurrent or metastatic castration-resistant prostate cancer: Canadian cancer trials Group Study IND205. Clin Genitourin Cancer 17: 201-208.e201, 2019.

47. Pitz MW, Eisenhauer EA, MacNeil MV, Thiessen B, Easaw JC, Macdonald DR, Eisenstat DD, Kakumanu AS, Salim M, Chalchal H, et al: Phase II study of PX-866 in recurrent glioblastoma. Neuro Oncol 17: 1270-1274, 2015.

48. Zhang N, Caenepeel S, Wang L, Norman M, Kendall R, Burgess T, Radinsky R, Hughes P and Freeman DJ: Abstract 2797: AMG 511, a potent and selective class I PI3K inhibitor, demonstrates anti-tumor activity in multiple xenograft models. Cancer Res 72: 2797-2797, 2012.

49. Fiascarelli A, Merlino G, Capano S, Paoli A, Bressan A, Bigion M, Scaltrit M, Arribas J, Bernadó Morales C, Pellacani A, et al: 1938P Characterization of the mechanism of action and efficacy of MEN1611 (PA799), a novel PI3K inhibitor, in breast cancer preclinical models. Ann Oncol: 30, 2019 doi: 10.1093/annonc/mdz268.065.

50. Speranza MC, Nowicki MO, Behera P, Cho CF, Chiocca EA and Lawler SE: BKM-120 (Buparlisib): A phosphatidyl-inositol-3 kinase inhibitor with anti-invasive properties in glioblastoma. Sci Rep 6: 20189, 2016.

51. Robert M, Frenel J, Bourbouloux E, Berton Rigaud D, Patsouris A, Augereau P, Gourmelon C and Campone M: Efficacy of buparlisib in treating breast cancer. Expert Opin Pharmacother 18: 2007-2016, 2017

52. Matulonis U, Wulf GM, Birrer MJ, Westin SN, Quy P, Bell-McGuinn K, Lasonde B, Whalen C, Aghajanian C, Solit DB, et al: Phase I study of oral BKM120 and oral olaparib for high-grade serous ovarian cancer (HGSC) or triple-negative breast cancer (TNBC). J Clin Oncol 32: 2510, 2014.

53. Matulonis UA, Wulf G, Barry W, Birrer M, Birrer M, Westin S, Spagnoletti T, Bell-McGuinn K, Obermayer E, Whalen C, et al: Abstract CT324: Phase I of oral BKM120 or BYL719 and olaparib for high-grade serous ovarian cancer or triple-negative breast cancer: Final results of the BKM120 plus olaparib cohort. Cancer Res 75: CT324, 2015.

54. Wang J, Li H, He G, Chu Z, Peng K, Ge Y, Zhu Q and Xu Y: Discovery of novel dual poly(ADP-ribose)polymerase and phosphoinositide 3-kinase inhibitors as a promising strategy for cancer therapy. J Med Chem 63: 122-139, 2020.

55. Owonikoko TK, Harvey RD, Carthon B, Chen Z, Lewis C, Collins H, Zhang C, Lawson DH, Alese OB, Bilen MA, et al: A Phase I study of safety, pharmacokinetics, and pharmacodynamics of concurrent everolimus and buparlisib treatment in advanced solid tumors. Clin Cancer Res 26: 2497-2505, 2020.

56. Bousmail D, Amrein L, Fakhoury JJ, Fakih HH, Hsu JCC, Panasci L and Sleiman HF: Precision spherical nucleic acids for delivery of anticancer drugs. Chem Sci 8: 6218-6229, 2017.

57. de Gooijer M, Zhang P, Buil LCM, Citirikkaya CH, Thota N, Beijnen JH and van Tellingen O: Buparlisib is a brain penetrable pan-PI3K inhibitor. Sci Rep 8: 10784, 2018.

58. Saura C, Bendell J, Jerusalem G, Su S, Ru Q, De Buck S, Mills D, Ruquet S, Bosch A, Urruticoechea A, et al: Phase Ib study of buparlisib plus trastuzumab in patients with HER2-positive advanced or metastatic breast cancer that has progressed on Trastuzumab-based therapy. Clin Cancer Res 20: 1935-1945, 2014.

59. Bavelloni A, Focaccia E, Piazzi M, Orsini A, Ramazzotti G, Cocco L, Blalock W and Faenza I: Therapeutic potential of nvp-bkm120 in human osteosarcomas cells. J Cell Physiol 234: 10907-10917, 2019

60. Müller A, Gillissen B, Richter A, Richter A, Chumduri C, Daniel PT and Scholz CW: Pan-class I PI3-kinase inhibitor BKM120 induces MEK1/2-dependent mitotic catastrophe in non-Hodgkin lymphoma leading to apoptosis or polyploidy determined by Bax/Bak and p53. Cell Death Dis 9 : 384, 2018.

61. Zhao P, Hall J, Durston M, Voydanoff A, VanSickle E, Kelly S, Nagulapally AB, Bond J and Saulnier Sholler G: BKM120 induces apoptosis and inhibits tumor growth in medulloblastoma. PLoS One 12: e0179948, 2017.

62. Lonetti A, Antunes IL, Chiarini F, Orsini E, Buontempo F, Ricci F, Tazzari PL, Pagliaro P, Melchionda F, Pession A, et al: Activity of the pan-class I phosphoinositide 3-kinase inhibitor NVP-BKM120 in T-cell acute lymphoblastic leukemia. Leukemia 28: 1196-1206, 2014 
63. Sun B, Jensen NR, Chung D, Yang M, LaRue AC, Cheung HW and Wang Q: Synergistic effects of SHP2 and PI3K pathway inhibitors in GAB2-overexpressing ovarian cancer. Am J Cancer Res 9: 145-159, 2019.

64. Yu F, Zhao J, Hu Y, Zhou Y, Guo R, Bai J, Zhang S, Zhang H and Zhang J: The combination of NVP-BKM120 with trastuzumab or RAD001 synergistically inhibits the growth of breast cancer stem cells in vivo. Oncol Rep 36: 356-364, 2016.

65. Chen IC, Hsiao LP, Huang IW, Yu HC, Yeh LC, Lin CH, Wei-Wu Chen T, Cheng AL and Lu YS: Phosphatidylinositol-3 kinase inhibitors, buparlisib and alpelisib, sensitize estrogen receptor-positive breast cancer cells to tamoxifen. Sci Rep 7: 9842, 2017.

66. Alipour F, Riyahi N, Safaroghli-Azar A, Sari S, Zandi Z and Bashash D: Inhibition of PI3K pathway using BKM120 intensified the chemo-sensitivity of breast cancer cells to arsenic trioxide (ATO). Int J Biochem Cell Biol 116: 105615, 2019.

67. Solberg NT, Waaler J, Lund K, Mygland L, Olsen PA and Krauss S: TANKYRASE inhibition enhances the antiproliferative effect of PI3K and EGFR inhibition, mutually affecting $\beta$-CATENIN and AKT signaling in colorectal cancer. Mol Cancer Res 16: 543-553, 2018.

68. Yao W, Yue P, Zhang G, Owonikoko TK, Khuri FR and Sun SY: Enhancing therapeutic efficacy of the MEK inhibitor, MEK162, by blocking autophagy or inhibiting PI3K/Akt signaling in human lung cancer cells. Cancer Lett 364: 70-78, 2015.

69. Blanco B, Herrero-Sanchez C, Rodriguez-Serrano C, Sanchez-Barba M and Del Canizo MC: Comparative effect of two pan-class I PI3K inhibitors used as anticancer drugs on human $\mathrm{T}$ cell function. Int Immunopharmacol 28: 675-685, 2015

70. Schimmer AD, Hedley DW, Penn LZ and Minden MD: Receptorand mitochondrial-mediated apoptosis in acute leukemia: A translational view. Blood 98: 3541-3553, 2001.

71. Pereira JK, Machado-Neto JA, Lopes MR, Morini BC, Traina F, Costa FF, Saad ST and Favaro P: Molecular effects of the phosphatidylinositol-3-kinase inhibitor NVP-BKM120 on T and B-cell acute lymphoblastic leukaemia. Eur J Cancer 51: 2076-2085, 2015.

72.Liu X, Li Q, Huang $\mathrm{P}$, Tong $\mathrm{D}, \mathrm{Wu} \mathrm{H}$ and Zhang $\mathrm{F}$ : EGFR-mediated signaling pathway influences the sensitivity of oral squamous cell carcinoma to JQ1. J Cell Biochem 119: 8368-8377, 2018

73. Bian X, Gao J, Luo F, Rui C, Zheng T, Wang D, Wang Y, Roberts TM, Liu P, Zhao JJ and Cheng H: PTEN deficiency sensitizes endometrioid endometrial cancer to compound PARP-PI3K inhibition but not PARP inhibition as monotherapy. Oncogene 37: 341-351, 2018.

74. Philip CA, Laskov I, Beauchamp MC, Marques M, Amin O, Bitharas J, Kessous R, Kogan L, Baloch T, Gotlieb WH and Yasmeen A: Inhibition of PI3K-AKT-mTOR pathway sensitizes endometrial cancer cell lines to PARP inhibitors. BMC Cancer 17: 638, 2017

75. Bashash D, Safaroghli-Azar A, Delshad M, Bayati S, Nooshinfar E and Ghaffari SH: Inhibitor of pan class-I PI3K induces differentially apoptotic pathways in acute leukemia cells: Shedding new light on NVP-BKM120 mechanism of action. Int J Biochem Cell Biol 79: 308-317, 2016

76. Preyat N and Leo O: Sirtuin deacylases: A molecular link between metabolism and immunity. J Leukoc Biol 93: 669-680, 2013

77. Bashash D, Delshad M, Riyahi N, Safaroghli-Azar A, Pourbagheri-Sigaroodi A and Momeny M: Inhibition of PI3K signaling pathway enhances the chemosensitivity of APL cells to ATO: Proposing novel therapeutic potential for BKM120. Eur J Pharmacol 841: 10-18, 2018.

78. Safaroghli-Azar A, Bashash D, Kazemi A, Pourbagheri-Sigaroodi A and Momeny M: Anticancer effect of pan-PI3K inhibitor on multiple myeloma cells: Shedding new light on the mechanisms involved in BKM120 resistance. Eur J Pharmacol 842: 89-98, 2019.

79. Zhang Y, Nie L, Xu K, Fu Y, Zhong J, Gu K and Zhang L: SIRT6, a novel direct transcriptional target of FoxO3a, mediates colon cancer therapy. Theranostics 9: 2380-2394, 2019.

80. Wang S, Niu X, Bao X, Wang Q, Zhang J, Lu S, Wang Y, Xu L, Wang $\mathrm{M}$ and Zhang J: The PI3K inhibitor buparlisib suppresses osteoclast formation and tumour cell growth in bone metastasis of lung cancer, as evidenced by multimodality molecular imaging. Oncol Rep 41: 2636-2646, 2019.
81. Trautmann M, Cyra M, Isfort I, Jeiler B, Krüger A, Grünewald I, Steinestel K, Altvater B, Rossig C, Hafner S, et al: Phosphatidylinositol-3-kinase (PI3K)/Akt signaling is functionally essential in myxoid liposarcoma. Mol Cancer Ther 18 834-844, 2019.

82. Jin L, Jin MH, Nam AR, Park JE, Bang JH, Oh DY and Bang YJ: Anti-tumor effects of NVP-BKM120 alone or in combination with MEK162 in biliary tract cancer. Cancer Lett 411: 162-170, 2017

83. Parrales A and Iwakuma T: Targeting oncogenic mutant p53 for cancer therapy. Front Oncol 5: 288, 2015.

84. Li Z, Xu X, Li Y, Zou K, Zhang Z, Xu X, Liao Y, Zhao X, Jiang W, Yu W, et al: Synergistic antitumor effect of BKM120 with Prima-1-met via inhibiting PI3K/AKT/mTOR and CPSF4/hTERT signaling and reactivating mutant P53. Cell Physiol Biochem 45: 1772-1786, 2018.

85. Aasen SN, Parajuli H, Hoang T, Feng Z, Stokke K, Wang J, Roy K, Bjerkvig R, Knappskog S and Thorsen F: Effective treatment of metastatic melanoma by combining MAPK and PI3K signaling pathway inhibitors. Int J Mol Sci 20: 4235, 2019.

86. Li M, Liang RF, Wang X, Mao Q and Liu YH: BKM120 sensitizes C6 glioma cells to temozolomide via suppression of the PI3K/Akt/NF-kappaB/MGMT signaling pathway. Oncol Lett 14: 6597-6603, 2017

87. De Martino D, Yilmaz E, Orlacchio A, Ranieri M, Zhao K and Di Cristofano A: PI3K blockage synergizes with PLK1 inhibition preventing endoreduplication and enhancing apoptosis in anaplastic thyroid cancer. Cancer Lett 439: 56-65, 2018.

88. Sai J, Owens P, Novitskiy SV, Hawkins OE, Vilgelm AE, Yang J, Sobolik T, Lavender N, Johnson AC, McClain C, et al: PI3K inhibition reduces mammary tumor growth and facilitates antitumor immunity and Anti-PD1 responses. Clin Cancer Res 23: 3371-3384, 2017

89. Anisuzzaman AS, Haque A, Wang D, Rahman MA, Zhang C, Chen Z, Chen ZG, Shin DM and Amin AR: In vitro and in vivo synergistic antitumor activity of the combination of BKM120 and erlotinib in head and neck cancer: Mechanism of apoptosis and resistance. Mol Cancer Ther 16: 729-738, 2017.

90. Yun MR, Choi HM, Kang HN, Lee Y, Joo HS, Kim DH, Kim HR, Hong MH, Yoon SO and Cho BC: ERK-dependent IL-6 autocrine signaling mediates adaptive resistance to pan-PI3K inhibitor BKM120 in head and neck squamous cell carcinoma. Oncogene 37: 377-388, 2017.

91. Liu X, Wu H, Huang P and Zhang F: JQ1 and PI3K inhibition synergistically reduce salivary adenoid cystic carcinoma malignancy by targeting the c-Myc and EGFR signaling pathways. J Oral Pathol Med 48: 43-51, 2019.

92. Sakakibara K, Tsujioka T, Kida JI, Kurozumi N, Nakahara T, Suemori SI, Kitanaka A, Arao Y and Tohyama K: Binimetinib, a novel MEK1/2 inhibitor, exerts anti-leukemic effects under inactive status of PI3Kinase/Akt pathway. Int J Hematol 110: 213-227, 2019.

93. Wang D, Li C, Zhang Y, Wang M, Jiang N, Xiang L, Li T, Roberts TM, Zhao JJ, Cheng H and Liu P: Combined inhibition of PI3K and PARP is effective in the treatment of ovarian cancer cells with wild-type PIK3CA genes. Gynecol Oncol 142: 548-556, 2016

94. Yang L, Yang G, Ding Y, Huang Y, Liu S, Zhou L, Wei W, Wang $\mathrm{J}$ and $\mathrm{Hu}$ G: Combined treatment with PI3K inhibitor BKM120 and PARP inhibitor olaparib is effective in inhibiting the gastric cancer cells with ARID1A deficiency. Oncol Rep 40: 479-487, 2018

95. Qiu Y, Pu T, Guo P, Wei B, Zhang Z, Zhang H, Zhong X, Zheng $\mathrm{H}$, Chen $\mathrm{L}, \mathrm{Bu} \mathrm{H}$ and Ye F: $\mathrm{ALDH}^{+} / \mathrm{CD} 44^{+}$cells in breast cancer are associated with worse prognosis and poor clinical outcome. Exp Mol Pathol 100: 145-150, 2016.

96. Wu L, Meng F, Dong L, Block CJ, Mitchell AV, Wu J, Jang H, Chen W, Polin L, Yang Q, et al: Disulfiram and BKM120 in combination with chemotherapy impede tumor progression and delay tumor recurrence in tumor initiating cell-rich TNBC. Sci Rep 9: 236, 2019.

97. Zhao H, Yang Q, Hu Y and Zhang J: Antitumor effects and mechanisms of olaparib in combination with carboplatin and BKM120 on human triple-negative breast cancer cells. Oncol Rep 40: 3223-3234, 2018

98. Yan G, Ru Y, Wu K, Yan F, Wang Q, Wang J, Pan T, Zhang M, Han H, Li X and Zou L: GOLM1 promotes prostate cancer progression through activating PI3K-AKT-mTOR signaling. Prostate 78: 166-177, 2017. 
99. Varambally S, Laxman B, Mehra R, Cao Q, Dhanasekaran SM, Tomlins SA, Granger J, Vellaichamy A, Sreekumar A, $\mathrm{Yu}$ J, et al: Golgi protein GOLM1 is a tissue and urine biomarker of prostate cancer. Neoplasia 10: 1285-1294, 2008.

100. Foster KA, Jane EP, Premkumar DR, Morales A and Pollack IF: NVP-BKM120 potentiates apoptosis in tumor necrosis factor-related apoptosis-inducing ligand-resistant glioma cell lines via upregulation of Noxa and death receptor 5. Int J Oncol 47: 506-516, 2015.

101. Radovich M, Solzak JP, Hancock BA, Conces ML, Atale R, Porter RF, Zhu J, Glasscock J, Kesler KA, Badve SS, et al: A large microRNA cluster on chromosome 19 is a transcriptional hallmark of WHO type A and AB thymomas. Br J Cancer 114: 477-484, 2016.
102. Bukum N, Novotna E, Morell A, Hofman J and Wsol V: Buparlisib is a novel inhibitor of daunorubicin reduction mediated by aldo-keto reductase 1C3. Chem Biol Interact 302: 101-107, 2019.

103. Hamid AR, Pfeiffer MJ, Verhaegh GW, Schaafsma E, Brandt A, Sweep FC, Sedelaar JP and Schalken JA: Aldo-keto reductase family 1 member C3 (AKR1C3) is a biomarker and therapeutic target for castration-resistant prostate cancer. Mol Med 18: 1449-1455, 2013.

104. Jiang ZB, Huang J, Xie C, Li X, Liu L, He J, Pan H, Huang L, Fan XX, Yao XJ, et al: Combined use of PI3K and MEK inhibitors synergistically inhibits lung cancer with EGFR and KRAS mutations. Oncol Rep 36: 365-375, 2016. 